\title{
Rechtsgeschichte
}

http://www.rg-rechtsgeschichte.de/rg6

Zitiervorschlag: Rechtsgeschichte Rg 6 (2005)

$\operatorname{Rg} 2005$

$192-195$

http://dx.doi.org/10.12946/rg06/192-195

\section{Thomas Vesting}

\section{Griechenlandsmirakel}




\section{Griechenlandsmirakel*}

Die Ausbildung des theoretischen Denkens in Griechenland vollzog sich nicht erst mit Platon und Aristoteles, sondern kündigte sich bereits im 6. Jh. an. Schon im archaischen Griechenland, in den frühen Ontologien der Vorsokratiker und bei den naturrechtlichen Vorstellungen der Sophisten, ist ein Fortschritt des Denkens in Richtung Rationalität erkennbar. Das ist der Ausgangspunkt der Untersuchung von Tobias Reichardt. Aber wie ist es zu dieser - im Vergleich zu allen anderen antiken Hochkulturen Vorderasiens einzigartigen Ideen-Evolution in Griechenland gekommen? Wie war das griechische Mirakel möglich? Auf diese Frage versucht Reichardt eine neue Antwort zu geben: Das theoretische Denken ging aus der neuen Organisationsform der polis und der darin eingelassenen Praxis der schriftlichen Gesetzgebung hervor. Dadurch gelang es erstmalig, die Ausdifferenzierung einer öffentlichen (Herrschafts-)Sphäre mit der Idee einer "guten Ordnung " als Leitgesichtspunkt allen politischen Handelns zu verknüpfen.

Diese tragende Bedeutung hat das Recht in Griechenland nicht von Anfang an. In der epischen Dichtung, bei Homer und Hesiod, lassen sich allenfalls Frühformen des Rechts erkennen: Insbesondere die Gerichtsszene im I 8. Buch der Ilias vermittelt das Bild eines noch geringen Grades politischer Vergemeinschaftung, in der sich höchstens Ansätze einer Polisbildung zeigen und eine »eigene Rechtssphäre " noch nicht existierte. Prédroit taucht allenfalls sporadisch in der "Gerichtssphäre « auf, in Form der Konfliktregulierung durch freiwillige Streitschlichtung. Die freiwillige Streitschlichtung verdrängte die Privatfehde und Blutrache aber keineswegs und blieb als Verfahren mehr oder weniger un- mittelbar an die Welt der Götter, der Mythen und der Divination angeschlossen. Auch weil alle Gerichtsoperationen noch auf das engste mit einer religiös interpretierten Tradition verwoben waren, insistiert Reichardt darauf, thémis und dike nicht als Bezeichnungen für ein schon existierendes Sinnsystem »Recht « zu interpretieren. Thémis kann also keinesfalls mit "Satzung ", dike keinesfalls mit "Recht « übersetzt werden. Vielmehr gehe es um Formeln für konkrete Streitfälle, die allesamt auf das Übliche, Gebräuchliche, Erlaubte referieren: auf das implizite Wissen um das Richtige, das nur ausgesprochen, nur gefunden, aber nicht »gesetzt « werden konnte. Bei Hesiod löst sich dike zwar ansatzweise vom konkreten Akt der Streitschlichtung, aber auch bei ihm gibt es noch keinen etwa vom Materiell-Nützlichen unterschiedenen Sinngehalt von »Gerechtigkeit «.

Von einem prédroit, welches sich selbst nur schwach oder kaum von anderen sozialen »Normen « unterschied und überdies religiös konnotiert blieb, führte erst der historisch relativ früh einsetzende Prozess der Einkerbung griechischer Stadtgesetze auf öffentlich zugänglichen Monumenten oder (Holz-)Stelen (stelai) weg. Reichardt beschränkt sich in seiner Darstellung der frühen Gesetzgebung auf Athen und zeigt zunächst, dass das Blutrecht Drakons zwar bereits den Anspruch auf "allgemeine Geltung " erhob, die Gesetze Drakons sich aber in die hergebrachte Ordnung einfügten. Mit Solon, so seine These, änderte sich dies von Grund auf. Solon tat nicht weniger, als die Bedingungen des politischen Lebens der Athener vollständig umzukrempeln: Einteilung der Bürger Athens in vier Einkommensklassen, die für Auf- und Abstieg

* Tobias Reichardt, Recht und Rationalität im frühen Griechenland (Contradictio Bd. 4), 234 S., Würzburg: Königshausen \& Neumann 2003, ISBN 3-8260-2649-7 
durchlässig waren; Verwandlung des Areopags in eine staatliche Institution, Schaffung des Rats der Vierhundert, Abschaffung der Schuldknechtschaft, Einführung der Popularklage und Überweisung von Verfahren an das Volksgericht sind einige der Errungenschaften, die Reichardt Solons Reformen zuschreibt. Das Ergebnis ist klar: Hier ging es nicht mehr um die gute alte Tradition, sondern im Gegenteil um einen "umfassenden und tiefgreifenden, kaum einen Gegenstandsbereich auslassenden und planmäßigen Eingriff in die überlieferte Ordnung «.

Solon erweist sich als ein früher - und über weite Strecken säkularer - politischer Denker. Seine moralische Integrität erlaubte es ihm, seinen Standpunkt der »Interesselosigkeit « in den politischen Prozess einzubringen. Dieser »objektive Standpunkt « führte zur Vorstellung einer neutralen Position der polis im Kampf der sozialen Interessen, wie er zugleich durch diese bedingt war. Dabei war es gerade das schriftlich festgehaltene Recht, mit dessen Hilfe es gelang, von den Sonderinteressen der Individuen und Gruppen zu abstrahieren und in der Eunomie, der "guten Ordnung «, die Interessendivergenzen aufzuheben. Damit glückte erstmalig eine planmäßige Gestaltung des »menschlichen Handlungsrahmens ", in der eine rationale »Dike-Vorstellung " praktisch wurde. Die thémistes und dikai der frühen Zeit wurden durch äußere, politische Planung zum Recht weiterentwickelt, und zwar mit dem Anspruch, eine "Gesamtordnung " herzustellen. Während das Denken Solons aber ein genuin politisches Denken blieb (wenn auch schon herausgelöst aus den Bindungen von Mythos und Tradition), wurde dieses autonome Denken in der Folgezeit zu einem autonomen philosophischen Denken ausgebaut. Das zeige sich, so Reichardt, vor allem daran, dass es das Recht der polis sei, nach dem nun auch andere Sachverhalte modelliert wurden. In der Vorsokratik wurden ontologische und kosmologische Notwendigkeit anhand der postulierten Notwendigkeit menschlichen Rechts vorgestellt, und Heraklit konnte den Widerspruch anhand von Vergleichen mit der Politik zu einem Prinzip des Seins erklären.

Reichardt hat eine dichte und gut geschriebene Untersuchung vorgelegt. Auch wenn der Duktus adornitischer »Herrschaftskritik «, der sich stellenweise einschleicht, inzwischen doch etwas anachronistisch anmutet, hebt sich sein entwicklungsgeschichtliches Denken, das politische Strukturen und politische Semantik miteinander in Beziehung setzt, wohltuend von vielen anderen, allzu geisteswissenschaftlichen Publikationen auf diesem Gebiet ab. Dass die polis auf dem Weg der Entfaltung theoretischen Denkens in Griechenland einen Beitrag geleistet hat, wird niemand, der sich auch nur ansatzweise mit der Geschichte der Antike befasst hat, bezweifeln wollen; G. Lloyd, J.-P. Vernant, M. Detienne u. a. haben dazu schon vor Reichardt eine Fülle von klugen Beobachtungen gemacht. Das große Problem indes, das Reichardts Variation dieses Themas hinterlässt, ist die Ausschließlichkeit, mit der das theoretische Denken aus den politisch-rechtlichen Verhältnissen abgeleitet wird. Dadurch avanciert die politisch-rechtliche Organisationsform des Stadtstaates zur "wesentlichen Bedingung « der Entstehung des rationalen Bewusstseins. Eine solche Rekonstruktion lässt sich jedoch kaum halten.

Reichardt begibt sich mit seinem Rekurs auf die frühe athenische Gesetzgebung auf äußerst glatten Boden. Insbesondere von den Solonischen Gesetzen ist kein Wort als Primärquelle erhalten, und ihre Authentizität kann heute keinesfalls mehr mit jener Selbstverständlichkeit unterstellt werden, wie Reichardt es letztlich 
tut. Reichardt unterschätzt in diesem Zusammenhang, wie sehr seit den Arbeiten von M. Parry, R. Carpenter und E. A. Havelock in der neueren Forschung die Rolle der oralen Traditionen und ihr überragender Einfluss bis in das 4. Jh. hinein akzentuiert wird. Es spricht vieles dafür, dass die Idee einer "umfassenden Gesetzgebung " frühestens um 4IO Gestalt annahm, nicht aber schon für die frühen Gesetzgebungen des 6. Jhs. unterstellt werden kann. ${ }^{\mathrm{I}}$ Reichardt vertraut allein auf Sekundärquellen, aber er übergeht die erhaltenen Hinweise darauf, dass die Solonischen Gesetze einer möglicherweise starken Interpolation durch Nichomachus am Ende des turbulenten 5. Jh. v. Chr. unterlagen. Das hat weitreichende Folgen: Die Eingriffe in das, was die Solonischen Gesetze gewesen sein könnten, resultierten nicht daraus, dass Teile zwischen dem 6 . und 4. Jh. verloren gegangen sein könnten, sondern aus der Unwahrscheinlichkeit, dass jemals mehr als einige Vorschriften von den Solon zugeschriebenen Gesetzen existierten. Robb hat die Sache auf den Punkt gebracht: »If only a small part of the regulations attributed to him were Solon's, Archaic Attica would have been the Hellenic version of George Orwell's I984. ${ }^{2}$

Deshalb ist die Annahme, dass es im archaischen Griechenland jemals eine Gesetzgebung mit dem Anspruch gegeben hat, eine »Gesamtordnung « zu verfassen, höchst problematisch. Dass es an einer systematischen Gesetzgebung fehlte, räumt Reichardt selbst mehrfach ein. Aber worin soll dann noch die Besonderheit der Solonischen Gesetzgebung bestanden haben? Die Gesetze konnten jetzt, da Schrift verwendet wurde, auf einen Urheber zurückgeführt, das Recht musste nicht mehr, wie unter Bedingungen primärer Oralität, als uraltes Recht von zeitloser Ewigkeit gedacht werden. Zweifellos wurde da- mit durch politisches Handeln (und Schriftgebrauch!) ein Kontingenzbewusstsein gegenüber der religiös interpretierten Tradition freigesetzt, ein Prozess der Zunahme von Voluntarismus in der Rechtsbildung (M. Weber) angeregt. Aber damit war kein umfassender und tief greifender, planmäßiger Eingriff in die überlieferte Ordnung verbunden. Nach allem, was man heute weiß, handelte es sich bei den Solonischen Gesetzen um punktuelle Lösungen konkreter politischer Konflikte. ${ }^{3}$

Schließlich fragt sich, was Reichardt überhaupt meint, wenn er von Recht spricht. Reichardt verwendet den Rechtsbegriff in einem äußerst weiten, Politik und Herrschaft einschließenden Sinn. Danach ist Recht nicht eine von Politik zu unterscheidende Sphäre, sondern Mittel der Realisation "gerechter Politik«. Kann man Recht aber auf seine politische Mobilisierung reduzieren, Recht also herrschaftssoziologisch kurzschließen? Diese noch heute, insbesondere unter Politologen weit verbreitete Vorstellung entstammt dem staatszentrierten Positivismus des I9. Jahrhunderts, für den Rechtsverbindlichkeit das staatliche Gewaltmonopol, ja selbst subjektive Rechte den Staat voraussetzen. Dieser Vorstellung hängt auch Reichardt an, aber es ist zu vermuten, dass man mit einem derartig staatsfixierten Rechtsbegriff weder dem modernen (liberalen) Recht noch der griechischen Realität gerecht wird. Das politikbedingte Kontingentwerden des Rechts blieb in Griechenland doch immer in die ontologische Struktur der Stadt als Raum der "politischen Identität " der Griechen eingebettet. Das ließ kaum eine Lösung des Stadtrechts von der Stadt als einer gegebenen politischen Ordnung zu. Aber selbst wenn man das Moment der Abstraktion vom Gegebenen stärker akzentuiert, hat das antike Griechenland doch allenfalls die Idee politischer

I Das belegen nicht nur die neueren

Arbeiten von H.-J. Hölkeskamp, auf die Reichardt knapp eingeht, sondern auch die feine Arbeit von K. Rовв, Literacy and Paideia in Ancient Greece, New York, Oxford I994, 99 ff., I 25 ff.

2 Roвb (Fn. I) I 28.

3 Vgl. nur Rовв (Fn. I) I 3 I f. 
Gerechtigkeit als Leitgesichtspunkt der städtischen Ordnung entdeckt und zu ihrer Bewahrung bestimmte Regeln und Grundsätze schriftlich als grammatá und später als nómoi fixiert. Aber dann bleibt immer noch die Frage unbeantwortet, was das spezifisch Rechtliche an der Idee politischer Gerechtigkeit und ihrer schriftlichen Fixierung gewesen sein soll?

Diese Frage stellt sich umso mehr, als die Gesetzgebungen ihrerseits niemals Gegenstand eines Denkens wurden, das nach der Einhaltung von Rechtsgrenzen bei der politischen Gesetzgebung gefragt hätte. Es gab in Griechenland politisches Denken und Gerichtsrhetorik, aber gerade kein Denken, das für eine rechtliche Grenzziehung Kriterien entwickelt und diese etwa bei Magistrats- oder Gerichtsentscheidungen zur Geltung gebracht hätte. Mit anderen Wor- ten: Es fehlte für den Bereich des Rechts gerade an einer Beobachtung zweiter Ordnung, die sich vorbehält noch zu prüfen, ob ein sich als rechtmäßig beschreibendes Handeln dies zu Recht oder zu Unrecht tut. Dass Solon seine Gesetzgebung als Teil der Herstellung einer "guten Ordnung « reflektierte, ist nur eine andere Formulierung dafür, dass die Griechen einen Beitrag zur Ausdifferenzierung des westlichen Politikbegriffs, nicht aber des Rechtsbegriffs geleistet haben. Reichardt kann mit seiner Untersuchung also allenfalls beweisen, dass die Politik die »wesentliche Bedingung « für die Entfaltung des theoretischen Denkens war. Ich würde auch dieser These widersprechen wollen, aber sie hätte dem Unternehmen Reichardts eine größere Konsistenz verliehen.

Thomas Vesting

\section{Russian Pandectistics meet Tolstoj*}

According to the author's own estimation, the reception of Roman law in Russia has still not been sufficiently investigated. He has decided to make his own contribution to this study by analyzing the academic biographies and legal views of the most noteworthy civilians of preRevolutionary Russia - Dmitrij Mejer, Nikolaj Djuvernua and Iosif Pokrovskij. Retrospectively, all these jurists seem the most prominent representatives of three successive generations of Russian academic lawyers. All of them had much in common in the character of their professional formation, being educated in Germany and therefore strongly influenced by German legal science in the theoretical background to their research activities. As the senior of them, Dmitrij
Mejer laid the foundations of the study of Russian civil law as a system elaborated on the basis of the application of the categories and logic of German Pandektenwissenschaft to the material of positive Russian law. His successor, Nikolaj Djuvernua, already worked within the framework of this normative mainstream, while the last of them, Iosif Pokrovskij, devoted himself to Roman law studies more than to the study of contemporary national law.

It seems that Avenarius has made an accurate selection of the individuals who may be considered representative for an understanding of the development of legal consciousness in late imperial Russia. He has tried to make them a medium for the understanding of this epoch in

\footnotetext{
Martin Avenarius, Rezeption

des römischen Rechts in Russland. Dmitrij Mejer, Nikolaj Djuvernua und Iosif Pokrovskij (Quellen und Forschungen zum Recht und seiner Geschichte, Bd. XI), Göttingen: Wallstein 2004, $80 \mathrm{~S}$., ISBN 3-89244-767-5
} 